\title{
Emerging Real-time Services: Optimizing Traffic by Smart Cooperation in the Network
}

\author{
Jose Saldana, Julián Fernández-Navajas, José Ruiz-Mas, University of Zaragoza \\ Dan Wing, Muthu A. M. Perumal, Michael Ramalho, Cisco Systems \\ Gonzalo Camarillo, Ericsson Research \\ Fernando Pascual, Diego R. Lopez, Manuel Núñez, David Flórez, Juan A. Castell, Telefonica I+D \\ Tomaso de Cola, Matteo Berioli, DLR (German Aerospace Center)
}

\begin{abstract}
The rise of new real-time interactive services, in which consumers demand a high subjective quality, makes it necessary to define new network mechanisms that can dynamically adapt to the traffic variations. Cooperation between the different actors is required in order to adapt the traditional network infrastructure to these new traffic patterns while guaranteeing delay requirements. Thus, standardization is needed in order to make cooperation possible. A method for Tunneling Compressed Multiplexed Traffic Flows (TCMTF) is proposed as an update to TCRTP, the current practice defined by the IETF for optimizing RTP flows. Some scenarios have been identified, in which traffic optimization can be deployed, while granting resource usage fairness and good user's experience. This proposal could be used in different application environments, as operators' networks, Internet service providers, long distance transports and game providers.
\end{abstract}

Keywords-multiplexing; header compression; real-time; standardization; tunneling; interactivity

\section{INTRODUCTION}

We are witnessing the emergence of real-time services that use the Internet for the delivery of interactive multimedia applications, available almost everywhere due to the success of smartphones and tablets. As an example of this significant growth, the mobile gaming market is expected to reach $\$ 54 \mathrm{~B}$ by 2015 [1]. The most common of these services is VoIP, but many others such as videoconferencing, telemedicine, video surveillance or online gaming are increasing their popularity. At the same time the number of wireless M2M (machine-to-machine) connections is steady growing since a few years, and a non-negligible (although exactly unknown) share of these is being used for delay-intolerant applications, e.g. industrial SCADA (Supervisory Control And Data Acquisition), power plant monitoring, smart grids, asset tracking. Taking into account the bandwidth scarcity of some of these scenarios, the design of architectures able to tightly integrate the corresponding dedicated infrastructures (e.g., sensor networks) with the terrestrial core network, so that services are seamlessly available to any consumer, is today a hot scientific topic, and part of the Internet of Things (IoT) paradigm.

Due to the specific requirements of these emerging services, their traffic presents a very specific profile, different from the normal traffic mix present on the Internet: long-term flows of small packets which are sent at a fast pace. However, taking into account that the first Internet architecture was designed for services without delay constraints (e.g., e-mail, file transfer, web browsing), it is not optimized for these emerging ones. This fact leads to some problems, as delay unpredictability, low efficiency in network usage, or congestion. The transition to IPv6, while having many advantages, will worsen this efficiency problem, since its overhead is bigger.

The use of architectures able to make use of network management and orchestration would provide many advantages, in terms of flexibility and robustness, since the optimization can be dynamically activated and its parameters tuned when required (e.g., traffic rush in a specific zone or moment). This saving would also benefit network stability, since service would be granted even when the traffic exceeds the normal parameters for which the network was designed. Automation and programmability of these functions and protocols are considered necessary in order to allow the network to react to the traffic status.

In addition to end consumers, other actors are concerned in these scenarios, and they should offer an optimal perception of the service: first, network operators, who have to accomplish these highly demanding requirements; next, service providers, who deploy the application and the supporting servers, but cannot control the path between them; network equipment developers who may include new features in their devices in order to correctly deliver these traffic flows; other concerned actors are enterprises with a number of connected central offices, satellite operators, and long transport carriers.

Sometimes, the collaboration between different actors will be required to optimize the traffic. Thus, standardization of the traffic optimization architecture is seen as essential in order to allow different entities to collaborate within a clearly defined shared framework. The architecture has to be flexible enough, in order to provide the possibility of optimizing traffic flows inside heterogeneous network technologies: Access Networks providing residential DSL or Broadband Fiber-to-thehome optical connections; MPLS networks of ISPs; broadband wireless systems, like LTE; backbone 
connections between Internet Routers of Internet Service Providers (ISPs), etc.

In order to permit the delivery of real-time content, RTP (Real-Time Protocol) was defined by the IETF. However, the definition of new protocols working over the same network, although necessary, is not enough. An additional effort is required in order to define new architectures and techniques, in order to add smartness and scalability to Future Networks, with the final aim of optimizing traffic, always guaranteeing an optimal quality of experience to the end user.

In particular, this work studies TCMTF (Tunneling Compressed Multiplexed Traffic Flows), a traffic optimization solution combining different protocols. When a number of flows using small packets traverse a common path segment, header compression [2] can be used to improve their efficiency. However, packets with compressed headers have to travel tunneled, so a multiplexing method is used to build a bigger packet including a number of payloads sharing the common tunnel header (Fig. 1). A demultiplexer is necessary at the end of the common path to rebuild the packets as they were originally sent, making multiplexing a transparent process for the ends of the communication. Tunneling also relieves intermediate routers from the decompression and compression process.

In the next section we summarize the traffic optimization techniques; next, we present in detail the suitable scenarios; finally some of the obtained results are summarized, in order to illustrate the benefits that can be obtained, while maintaining subjective quality.

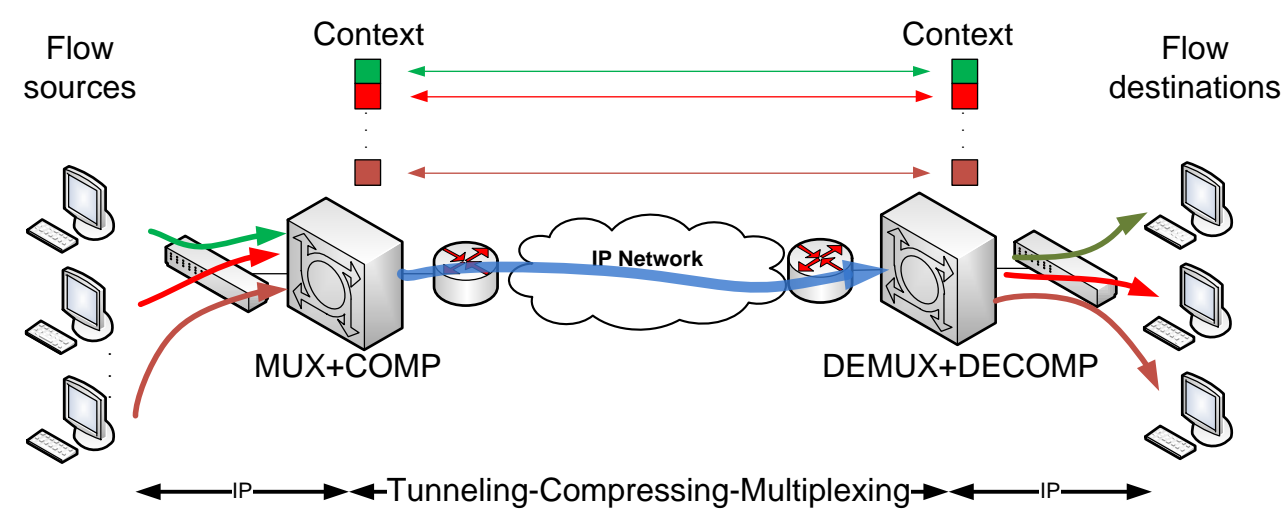

Figure 1. Tunneling, Compressing and Multiplexing Traffic Flows (TCMTF) scheme

\section{OPTIMIZATION TECHNIQUES}

In order to better understand our optimization proposal, this section summarizes the state of the art about traffic optimization by means of header compression, multiplexing and tunneling, with a special emphasis on the need of preserving the interactivity of the optimized traffic flows, and the end user's perception in turn.

\section{HEADER COMPRESSION}

Different header compression techniques have been defined in the last several years by the IETF [2]. The first one was developed in 1990, when Van Jacobson defined VJHC, which was able to jointly compress TCP/IP headers. Later, IPHC was defined, as a standard also able to compress UDP and IPv6 headers. At the same time, cRTP (compressed RTP) was developed, being able to compress RTP headers, too.

These techniques are based on the fact that many header fields are the same for every packet in a flow. Another improvement is the use of delta compression so as to reduce the number of bits required by a field, by transmitting only the difference between the value of a field in a packet and in the previous one. Bandwidth can be saved, but at the cost of defining a context, which is a set of variables that has to be synchronized between the sender and the receiver.

In 1999, there was a desire to send voice over an IP network with bandwidth efficiency on par with voice over ATM. The already existing cRTP could not accommodate significant delay, reordering or packet loss, so it was extended to support such network characteristics, and standardized as ECRTP (Enhanced cRTP). When operating on routers (rather than on endpoints themselves), ECRTP needs to be tunneled.

\section{RTP TUNNELING-COMPRESSING AND MULTIPLEXING}

In the late 90's, the IETF felt the need of standardizing some multiplexing method that could be able to reduce bandwidth in RTP flows. The group discussed different proposals and finally approved a traffic optimization standard including an RTP compressing protocol, a multiplexing one and a tunneling one (RFC 4170, "TCRTP"). The document was approved as "Best Current Practice" since it did not define any new protocol, but it only established a way to combine them: first, it runs ECRTP over PPPMux to gain the ability to multiplex several ECRTP payloads into one packet. Finally, this is all run over an L2TP tunnel over IP. 
After the standardization of TCRTP, there has been additional interest on header compression. In 2006, the IETF formed the Robust Header Compression (ROHC) Working Group, which created new specifications, with a special care of context synchronization. For most RTP flows, ROHC is more efficient than ECRTP, at the cost of implementation complexity [2]. Once the effort for designing $\mathrm{ROHC}$ has been conducted, it is worth including it in the tunneling, compressing and multiplexing standard.

\section{WIDENED PROPOSAL}

In the past few years, many real-time interactive applications that do not use RTP have become popular. Some of them send bare UDP packets, e.g., First Person Shooter (FPS) online games [3], for which latency is very critical [4]. There is also another fact which has to be taken into account: TCP is used for streaming media delivery: for several reasons, such as avoiding firewalls, the standard RTP is substituted in many cases by other options using HTTP.

Furthermore, there is another kind of applications that have been reported as real-time using TCP [5]: MMORPGs (Massively Multiplayer Online Role Playing Games), which in some cases have millions of players [6], thousands of them sharing the same virtual world. They use TCP packets to send the player's commands to the server, and also to send to the player's application the characteristics and situation of other gamers' avatars. These games do not have the degree of interactivity of FPSs, but the quickness and the movements of the player are important, and may decide if they win or lose a fight.

Recent research [7], [8] has highlighted the usefulness of these optimization techniques for nonRTP flows. Taking these results into account, the contact between researchers from academia and industry has led to the proposal of a new standard widening the spectrum of the current multiplexing, compressing and tunneling scheme, so as to consider not only RTP flows, but also UDP and even TCP ones. New scenarios of application have been proposed, and some of them have been studied using suitable tools in order to evaluate the savings that can be expected. The results can be beneficial for equipment developers, long distance transports (transoceanic wires and satellite service providers) and network operators.

Another characteristic of these services is that the aggregated traffic does not present a stationary behavior (e.g., the release of a new game, different hours of the day, weekends, a crowded spot in the mobile environment). In this sense, traffic optimization can provide flexibility when dealing with "rush hours". The network may not be dimensioned for these cases, but a number of flows can be multiplexed together, thus slightly reducing the quality, but avoiding the blocking of the service by means of bandwidth saving.

\section{EFFECT ON SUBJECTIVE QUALITY}

On the other hand, a concern for some of the actors in this scenario (e.g., network operators) is the counterpart of these multiplexing techniques: although they reduce bandwidth consumption, they introduce additional impairments, which may be critical for certain services. As an example, the consumers of online games show a very demanding profile [9], so traffic savings cannot be obtained at the cost of a significant reduction of subjective quality.

As a counterpart of traffic optimization, small additional delays may appear, since the multiplexer will have to retain packets in order to group a number of them. Second, an extra jitter will also be added, since some packets will spend more time than others at the multiplexer's queue. Finally, the packet size will be increased, and this may also have an influence on packet loss, as shown in [10].

For this aim, subjective quality estimators for different services [11], [12], [13] have been used in order to translate multiplexing delay into subjective quality score reduction. Research studies have obtained the maximum delay that the consumers may tolerate. Although out of the scope of the current paper, they can be found in [8], [10], [14]. Taking into account that multiplexing period can be dynamically tuned according to the status of the network, it has been shown that it is possible to optimize the traffic while not annoying the users [7].

\section{SCENARIOS OF APPLICATION}

Different scenarios of application can be considered for TCMTF architecture. As we will next explain, different actors are involved, and may obtain a benefit of traffic compression: network operators, application providers, device manufacturers, companies with a number of connected central offices, satellite operators, long transport carriers, etc. As we will see, even the final consumer can perform optimization and get savings in some cases.

\section{RESIDENTIAL SCENARIO}

As a first example, we can consider the residential users of a real-time interactive application (e.g., an online game generating small packets) in a town or a district. Here, a compressing and multiplexing module can be included in network devices, in order to group packets with the same destination (Fig. 2a). Depending on the number of users of the application, the packets could be grouped at different levels in DSL fixed network scenarios, at gateway level in LTE mobile network scenarios or even in other ISP edge routers. Although not shown in the figure, TCMTF may also be applied for fiber residential accesses, and in $2 \mathrm{G} / 3 \mathrm{G}$ mobile networks. This would reduce bandwidth requirements in the provider aggregation network, and in the network connection of the application provider, thus resulting in savings for both actors. 
In this scenario, agreements between different companies can be established in order to save bandwidth and to reduce packets per second. For example, a service provider (e.g., an online gaming enterprise) could be allowed to place a multiplexer in the aggregation network of an ISP, being able to group all the flows of a game or service. Another server would demultiplex these packets once they arrive to the network of the provider.

At the same time, the ISP would implement TCMTF capabilities within its own MPLS network in order to optimize internal network resources: compressing and multiplexing modules could be embedded in the Label Edge Routers of the network. In that scenario MPLS would be the "tunneling" layer, being the tunnels the paths defined by the MPLS labels and avoiding the use of other tunneling protocols.

\section{CORPORATE ENVIRONMENTS}

Not only network operators can deploy traffic optimization, but also end users can compress traffic end-to-end from network borders. As an example, we can consider the case of an enterprise with a number of distributed central offices, in which an appliance could be placed next to the access router, being able to multiplex and compress traffic flows with a shared origin and destination (Fig. 2b). Thus, a number of remote desktop sessions to the same server could be multiplexed, or a number of VoIP calls between two offices could also require less bandwidth and fewer packets per second.

Another example of an end user collaborating in traffic optimization could be an Internet café, which is suitable of having many users of the same application (e.g., a game) sharing the same access link. Internet cafés are very popular in countries with relatively low access speeds in households, where home computer penetration is usually low as well. In many of these countries, bandwidth can become a serious limitation for this kind of business, so TMCTF savings may become critical for their viability.

End-to-end optimization can also be interesting for satellite communication links, which often manage the bandwidth by limiting the transmission rate, measured in packets per second, to and from the satellite. Applications like VoIP that generate a large number of small packets can easily fill the limited number of pps slots, limiting the throughput across such links. This results in poor utilization of the satellite link's bandwidth as well as places a low cap on the number of voice calls that can utilize the link simultaneously. Thus, multiplexing small packets into one packet for transmission would improve the efficiency. It would also be useful to multiplex small TCP packets into one packet - this could be especially interesting for compressing TCP ACKs.

\section{MACHINE TO MACHINE (M2M) SCENARIO}

Finally, an additional scenario for TCMTF is the transport over a satellite link of M2M/SCADA services (Fig. 2c), which may include tracking and tracing, asset monitoring, metering, security and video surveillance, just to cite a few. M2M terminals are themselves equipped with sensing devices and, more interestingly, can interface to proximity sensor networks through wireless interfaces (e.g., Wi-Fi, ZigBee, Bluetooth). A number of agents (devices, Wireless Sensor Networks, etc.) generate messages to be distributed to the data centers via a satellite link, accessed through satellite terminals. Resource scarcity is characteristic of these specialized links, so they usually include advanced means of optimization. However, once the traffic is received at the gateway connecting the satellite link to the Internet, it is fed into it without any sort of optimization, which could impact in the overall performance.

In this context, TCMTF optimization deployed at the gateway ending the satellite link can optimize the traffic, thus avoiding network capacity wastage, by setting up tunnels to the Data Centers. If the case of large sensor deployments is considered, where proximity sensor networks transmit data through different satellite terminals, the use of compression algorithms to reduce the overhead introduced by TCP or UDP and IPv6 protocols is certainly desirable.

All in all, the development of a new standard with a wider scope seems necessary, and can be interesting for many different players, often in a collaborative way: developers of VoIP systems can include this option in their solutions, or game providers can achieve bandwidth savings in their supporting infrastructures. Other fact that has to be remarked is that the technique not only saves bandwidth but also reduces the number of packets per second, which sometimes can be a bottleneck for a satellite link or even for a mid or lowend network router [3]. 
Home network with a number of users (e.g. Internet Café, access shared by neighbours)
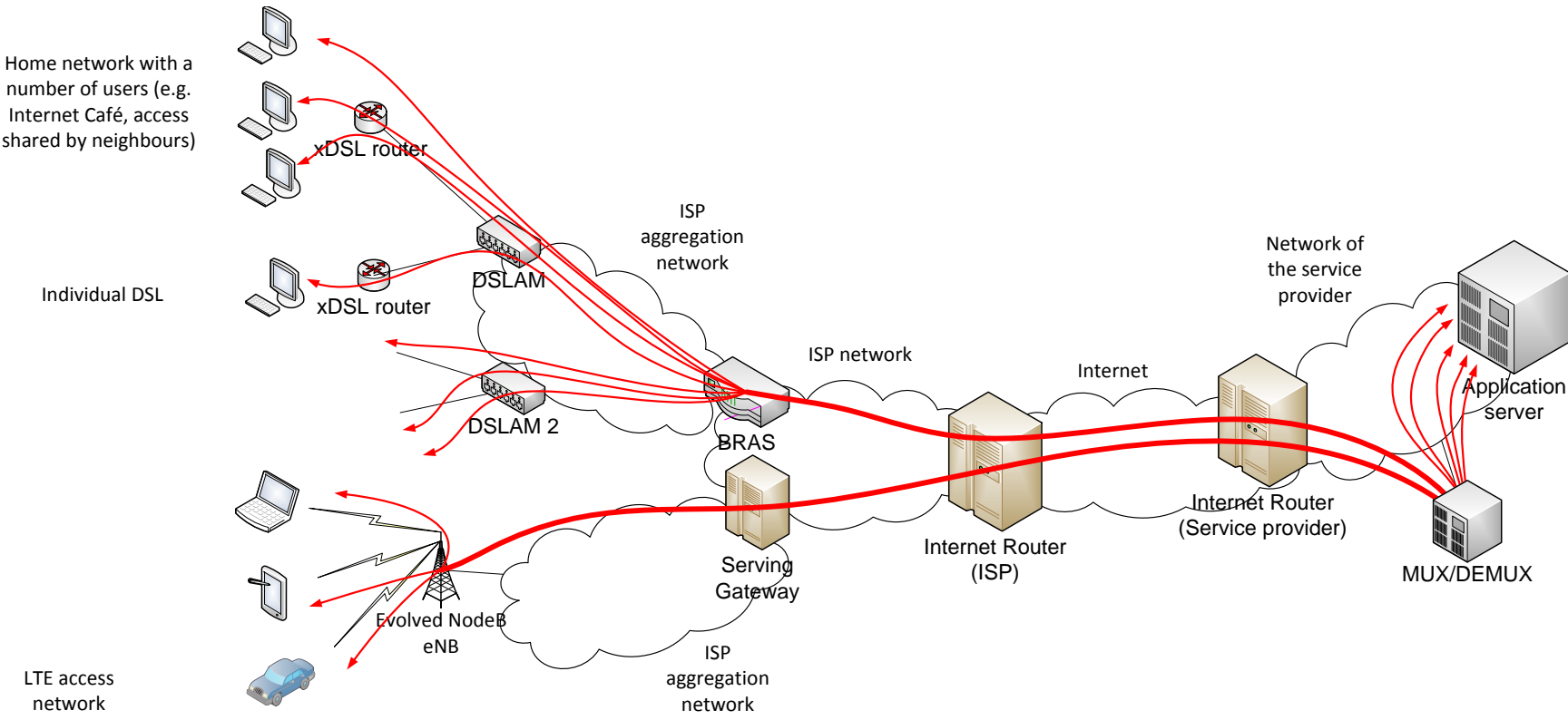

(a)

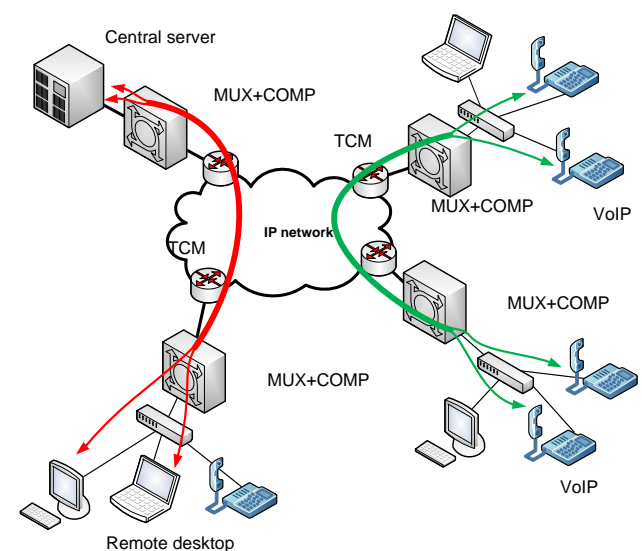

(b)

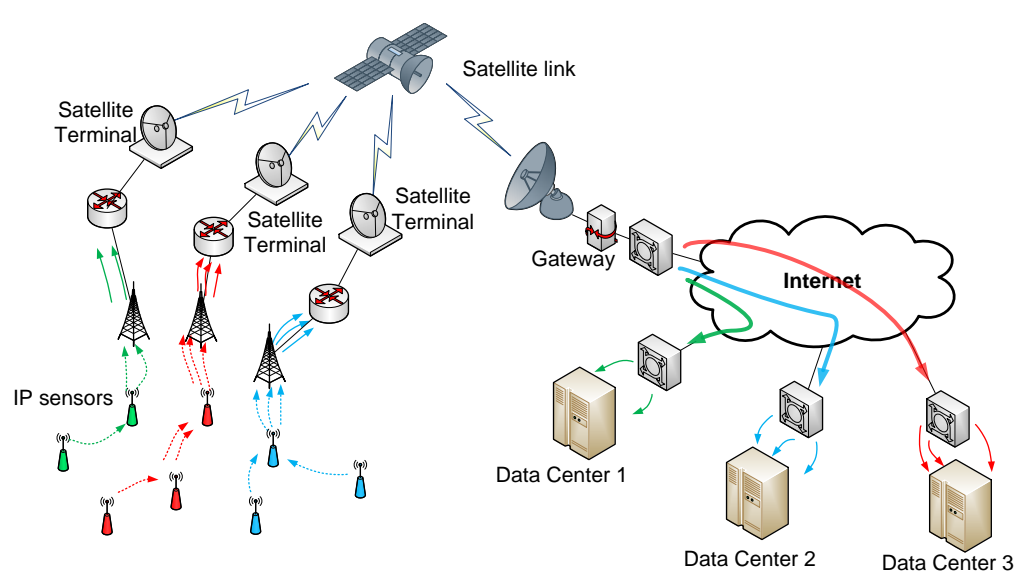

(c)

Figure 2. Traffic optimization scenarios: a) multiplexing flows of the same ISP in a residential scenario; b) appliance between central offices in a corporate scenario; c) sensor network sending traffic via a satellite link

\section{Emerging Services' Traffic OPTimization}

In this section we present some examples of the improvements that can be obtained by the optimization proposal. Research studies using UDP [7], TCP [8] and RTP [10] traffic have remarked these savings.

Fig. 3 presents a real-scale graphical representation of packet sizes of native and TCMTF compressed flows, for the services used as an example in this section. It can be observed that the native traffic presents a high overhead, caused by the small size of the payload. However, when multiplexing, the common overhead is shared by a number of packets, thus reducing the overhead per packet.

In each of the examples, we will separately present an IPv4 and an IPv6 case. As we will see, in the case of
IPv6, the bandwidth savings are higher, since the header is twice as big as the one of IPv4, but it can be compressed to a similar number of bytes: header compression avoids the sending of the fields which are the same for every packet, so IPv6 origin and destination addresses can be avoided totally.

As highlighted in [7], a suitable policy has to be defined in order to select the native packets to be included on each multiplexed one. Since many of the considered services do not use a fixed cadence [15], a multiplexing period is defined in order to place in the same bundle all the packets arrived during the interval. Fig. 4 and Fig. 5 present the results of bandwidth saving and packets per second respectively. Bandwidth savings have been measured as the quotient of optimized bandwidth divided by native one. For the 
sake of simplicity, Fig. 4 only shows the results for IPv4. Some IPv6 results can be found in the cited references.

\section{RTP-BASED SERVICE}

As a first example, we will present some VoIP measurements that were carried out in [10]. Although they are valid for both the current standard (TCRTP) and TCMTF, we think presenting them is interesting in order to compare their savings with the ones obtained for other services. First Fig. 3a) shows the optimization of G.729 flows with two samples per packet ( 20 bytes of payload). We can see that in this case header reduction is significant. The cause of this is that we can compress IP/UDP/RTP headers, which are 40 bytes for $\mathrm{IPv} 4$, and 60 for IPv6.

Fig. 4a) shows that significant savings (above 60 percent in some cases) can be achieved when multiplexing different numbers of voice flows, depending on the number of samples per packet $(1,2$ or 3 samples, which means 10,20 or 30 bytes of payload if G.729 codec is used). The results are presented in this case as a function of the number of flows, since we are considering that all the calls use the same codec. In this case we can use a multiplexing period equal to interpacket time, thus having one packet of each flow in the multiplexed bundle and reducing the added delay. If flows using different codecs were grouped together or if a variable rate codec was used, a suitable multiplexing period should be defined in order to avoid excessive delay and jitter. It can be seen in the figure that the saving presents an asymptote, which implies that the number of merged flows cannot be increased indefinitely.

Regarding the reduction of packets per second, Fig. 5a) shows that their amount can be significantly reduced: if 50 RTP flows are multiplexed, we may pass from 5,000 to less than 300 packets per second.

\section{UDP-BASED SERVICE}

As an example of the gaining that can be achieved for services using UDP (but not RTP) packets, we present the case of a FPS game (Counter Strike 1). Fig. $3 \mathrm{~b}$ ) shows a comparison between native and optimized packets. In Fig. 4b) the bandwidth saving for the clientto-server traffic is presented as a function of the number of players (i.e. number of flows) and the multiplexing period. As the payload is bigger than in the case of VoIP, the bandwidth saving is smaller (about 30 percent). A comparative study including eight different FPS games can be found in [7]. In some cases, the bandwidth saving for IPv6 was above 50 percent. A study of the impact of multiplexing on the subjective quality of an FPS can be found in [14].

The reduction in terms of packets per second is also significant in this case (Fig. 5b): it can be observed that, in the case of 20 players, we may pass from almost 500 to less than 20 packets per second, thus alleviating the workload of routers and network infrastructure.
As an additional example, the saving obtained in an Industrial application that could be carried in a M2M service is shown in Fig. 3c). In this case, only the IPv6 case is shown, since it is commonly used in this context.

\section{TCP-BASED SERVICE}

Finally, as an example of a TCP-based service, we present some tests that have been deployed using the traffic of the most popular MMORPG nowadays (World of Warcraft) [8]. As it can be seen in Fig. 3d), the use of TCP instead of UDP is important, since there are a high number of ACK packets without payload. In this case, header compression is applied to the whole packet, thus obtaining a very high compression rate. It can be observed that savings higher than the ones obtained for FPSs can be achieved: 60 percent of the bandwidth can be saved for IPv4, and this figure reaches 70 percent if IPv6 is used.

On the other hand, we see that the period and the number of players have to be higher in order to be near the asymptote. However, this is not a problem, since the interactivity of these games is not as critical as in FPSs, so higher values of the period can be used while maintaining an acceptable subjective quality [8], [12]. Regarding the number of players, in these games the virtual scenario can be simultaneously shared by thousands of them, whereas in FPSs this number is usually limited to 20 or 30 .

Finally, it must be noticed that the amount of packets per second is also reduced significantly (Fig. 5c). For the case of multiplexing the traffic of 100 players, we improve from sending more than 900 to less than 10 packets per second.

\section{Proposal of a Widened STANDARd}

The contact between academy researchers and the industry has permitted the first steps toward the formal definition of this proposal, which was first presented in the context of the Transport Area Working Group (tsvwg) in the IETF 83 meeting in Paris (March 2012). The main idea was to make it cover a wider scope, being able not only to save bandwidth for RTP, but also for real-time interactive flows using other protocols. The $\operatorname{draft}^{1}$ is being discussed on a specific mailing list (tcmtf@ietf.org). The current version includes the use cases explained in this paper. However, new ones are expected to be included during the next steps.

Fig. 6 shows the protocol stack of the current standard (TCRTP) (a), and also the new proposal (b), which considers different possibilities for real-time traffic. As it can be seen, the new proposal is backwards compatible, since it includes all the options considered in the current standard.

\footnotetext{
${ }^{1}$ https://datatracker.ietf.org/doc/draft-saldana-tsvwg-tcmtf/
} 
Five IPv4/UDP/RTP VolP packets with two samples of 10 bytes $\eta=100 / 300=33 \%$

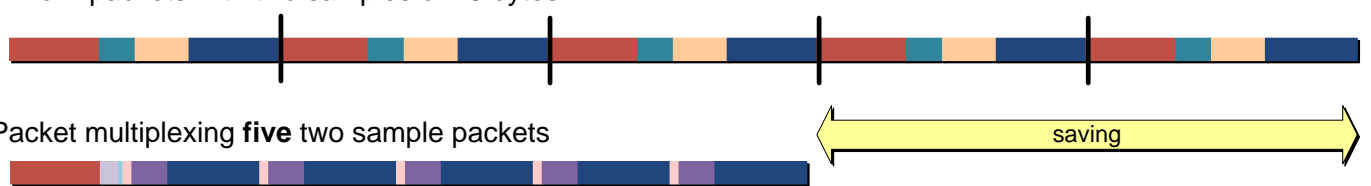

One IPv4 TCMTF Packet multiplexing five two sample packets $\eta=100 / 161=62 \%$

Four IPv6/UDP/RTP VolP packets with two samples of 10 bytes $\eta=80 / 240=33 \%$

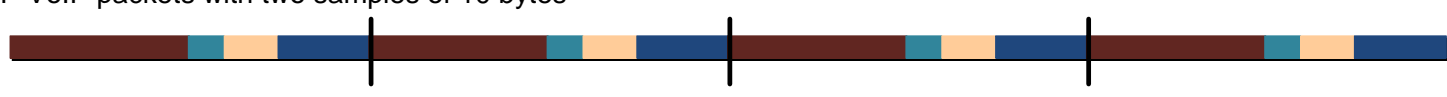

One IPv6 TCMTF Packet multiplexing four two sample packets $\eta=80 / 161=49 \%$

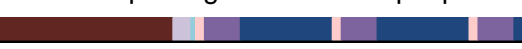

(a)

Four IPv4/UDP client-to-server packets of Counter Strike $\eta=244 / 356=68 \%$

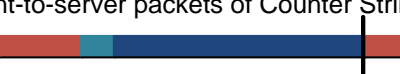

One IPv4/TCMTF packet multiplexing four client-to-server Counter Strike packets $\eta=244 / 293=83 \%$

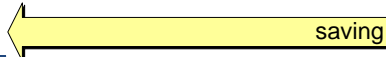
saving

Three IPv6/UDP client-to-server packets of Counter Strike $\eta=183 / 327=56 \%$

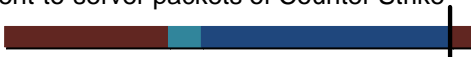

One IPv6/TCMTF packet multiplexing three client-to-server Counter Strike packets $\eta=183 / 246=74 \%$

Four IPv6/UDP Packets. Flow monitoring in industrial applications. Payload: 40 bytes $\eta=160 / 352=45 \%$ \begin{tabular}{l|l|l|} 
& \\
\hline
\end{tabular}

One IPv6/TCMTF packet multiplexing four packets for industrial applications $\eta=160 / 223=71 \%$

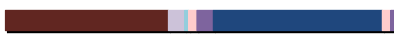

(c)

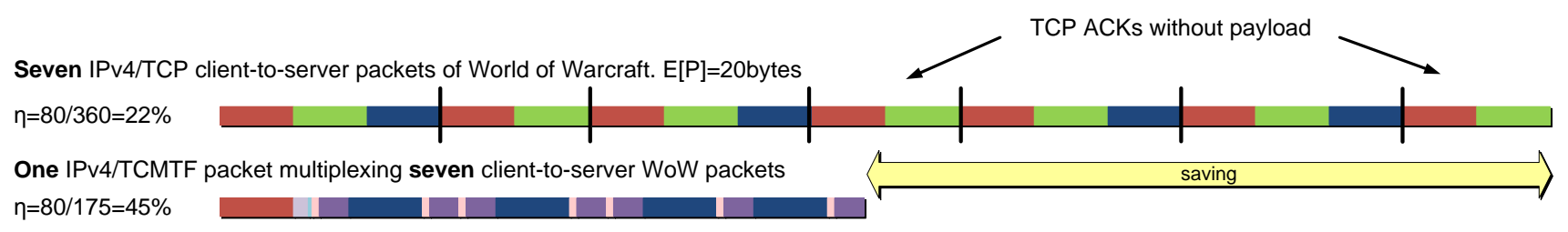
$\eta=80 / 175=45 \%$

Five IPv6/TCP client-to-server packets of World of Warcraft. $E[P]=20$ bytes $\eta=60 / 360=16 \%$

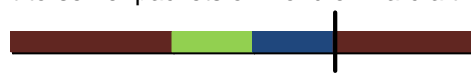

One IPv6/TCMTF packet multiplexing five client-to-server WoW packets $\eta=60 / 187=32 \%$

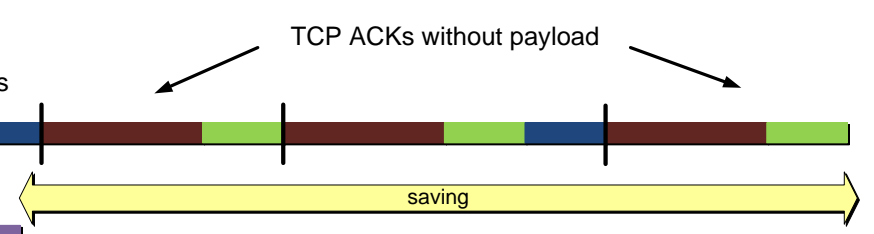

(d)

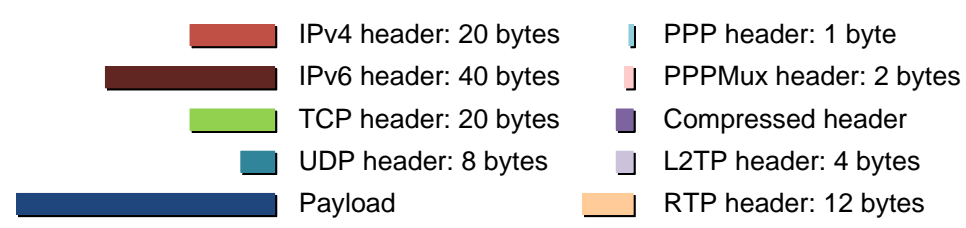

Figure 3. Scheme of the original and TCMTF packets for IPv4 and IPv6, for a) VoIP; b) a FPS game (Counter Strike I); c) Monitoring flow in M2M industrial applications; d) an MMORPG game (World of Warcraft) 


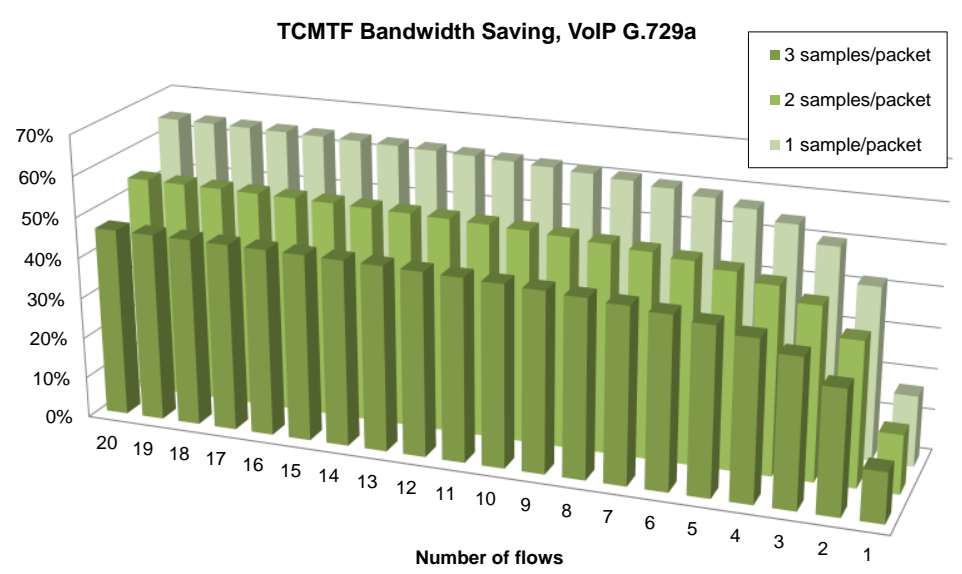

(a)

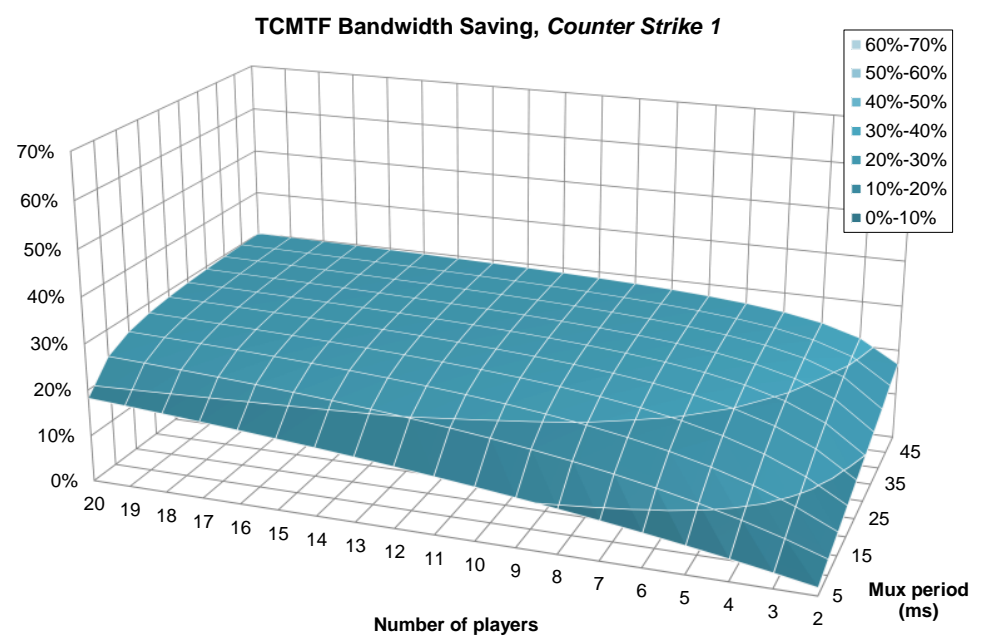

(b)

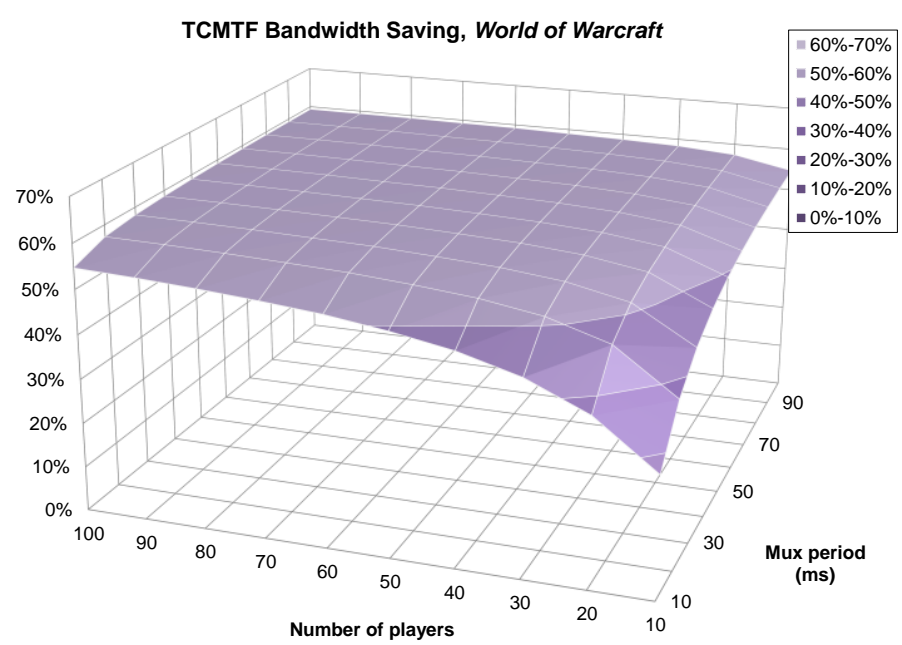

(c)

Figure 4. Bandwidth saving using TCMTF with IPv4 for a) G.729 VoIP flows; b) Counter Strike 1; c) World of Warcraft

Regarding compression, a number of options are considered: as different standards are able to compress various headers, the one to be used could be selected depending on the traffic to compress, and taking into account the availability of processing and memory resources. In addition, the proposal also considers the possibility of having null header compression, for clients that do not want to compress traffic, taking into account the need of storing a context for every flow and the problems of context desynchronization in certain scenarios. Further analysis will be required in order to study the tradeoffs between compressing rate and processing and memory requirements in each case. 


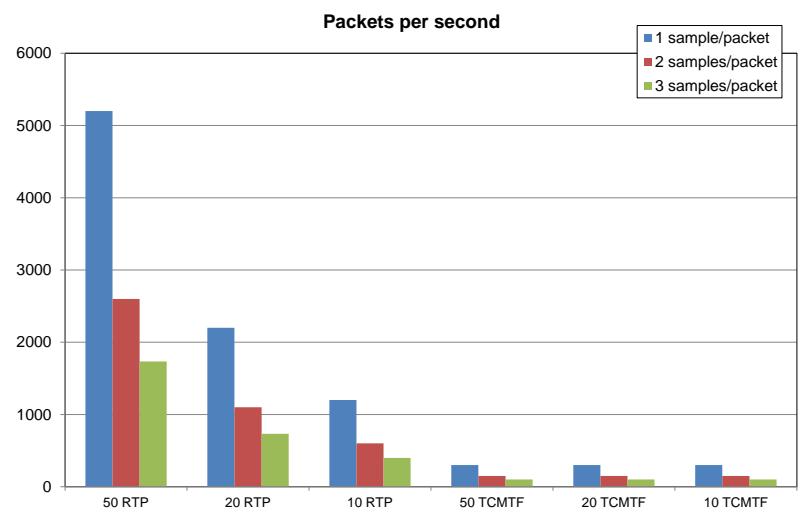

(a)

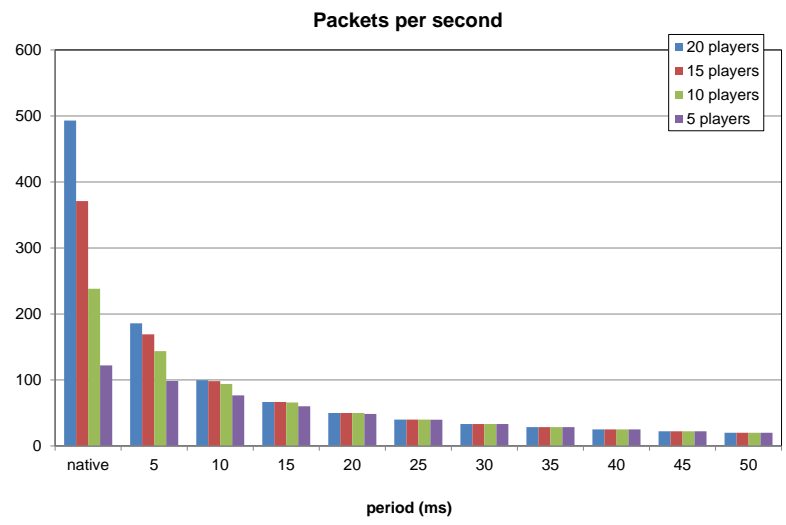

(b)

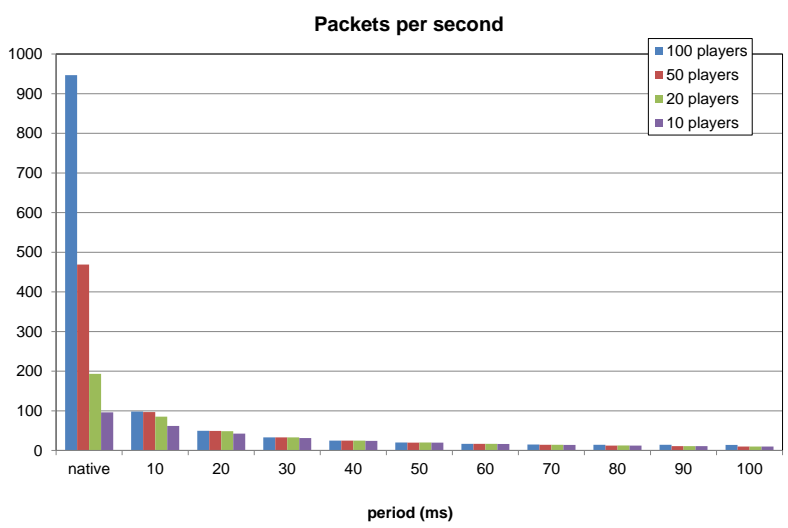

(c)

Figure 5. Packets per second saving using TCMTF for a) G.729 VoIP flows; b) Counter Strike 1; c) World of Warcraft

With respect to multiplexing and tunneling, TCRTP uses PPPMux and L2TP, both of which are relatively complex protocols. Simpler mechanisms could also be considered as a possibility but it would have to be newly defined. GRE (RFC 2784) and a lightweight multiplexing mechanism are appealing.

There is another topic that has to be considered: although VoIP packets are usually generated at a fixed rate, in other services inter-packet time does vary. So we have to define a policy to decide which packets are multiplexed and when. As research has shown, each service has a maximum delay tolerated by the users, so an upper bound for the added delay and jitter has to be set, in order not to harm the subjective quality. Therefore, multiplexing policies based on a period are considered the most adequate. As reported in [14], network propagation delay has to be taken into account too: the smaller the network propagation delay, the bigger the available margin for multiplexing delay.

The mechanisms necessary to establish the tunnel between endpoints will have to be defined by the standard, and also the negotiation protocol, which could be used in order to decide the option to use in each layer.

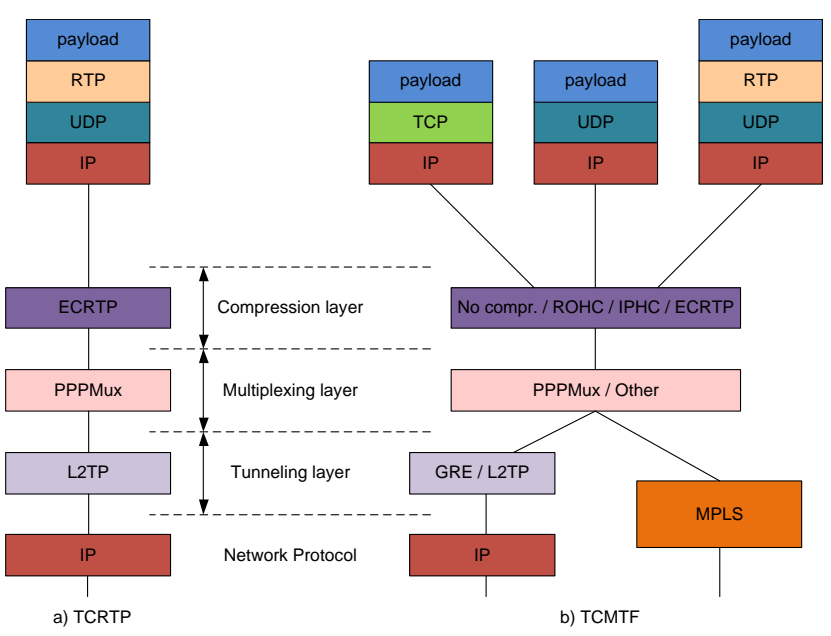

Figure 6. Protocol stack of a) TCRTP and b) TCMTF

\section{ConCLUSION}

In this article the problem of improving bandwidth efficiency in real-time interactive services has been first highlighted, identifying different scenarios of application in which a number of flows share a common path segment. By the use of multiplexing, tunneling and header compression, bandwidth can be saved not only for RTP flows but also for other real-time interactive services. The studied scenarios include aggregation networks of ISPs, tunnels connecting central offices of an enterprise or satellite links in M2M/SCADA environments.

The obtained savings for real-time applications in these scenarios, and the need for collaboration between the different actors show the convenience of updating the current standard. As a result, a proposal for widening its scope has been presented, including different possibilities for traffic flows, compressing and tunneling protocols.

\section{ACKNOWLEDGMENTS}

This work has been partially financed by CPUFLIPI Project (MICINN TIN2010-17298), Aragon Government and European Social Fund, and Catedra Telefonica, Univ. Zaragoza. 


\section{REFERENCES}

[1] Juniper Research: Whitepaper "Entertainment on the move! Markets, Opportunities \& Forecasts 2011-2015".

[2] E. Ertekin, C. Christou, "Internet protocol header compression, robust header compression, and their applicability in the global information grid," IEEE Communications Magazine, vol. 42, 2004, pp. 106-116.

[3] W. Feng, F. Chang, W. Feng and J. Walpole, "A Traffic Characterization of Popular On-Line Games," IEEE/ACM Trans. Networking, pp. 488-500, 2005.

[4] S. Zander, G. Armitage, "Empirically Measuring the QoS Sensitivity of Interactive Online Game Players," Australian Telecommunications Networks \& Applications Conference 2004 (ATNAC2004), Sydney, Australia, Dec. 2004.

[5] P. Svoboda, W Karner, M. Rupp, "Traffic Analysis and Modeling for World of Warcraft," ICC '07. IEEE International Conference on Communications, pp.1612-1617, 24-28, Jun. 2007.

[6] K. Chen, P. Huang, C. Lei, "Game traffic analysis: An MMORPG perspective," Proc. international workshop on Network and operating systems support for digital audio and video (NOSSDAV'05), pp. 19-24. ACM, New York, 2005.

[7] J. Saldana, J. Fernández-Navajas, J. Ruiz-Mas, J. I. Aznar, E. Viruete, L. Casadesus, "First Person Shooters: Can a Smarter Network Save Bandwidth without Annoying the Players?," IEEE Communications Magazine, Consumer Communications and Networking Series, vol. 49, no. 11, pp. 190-198, Nov. 2011.

[8] J. Saldana, L. Sequeira, J. Fernandez-Navajas, J. Ruiz-Mas, "Traffic Optimization for TCP-based Massive Multiplayer Online Games," Proc. International Symposium on Performance Evaluation of Computer and Telecommunication Systems SPECTS 2012, July 811, 2012, Genoa, Italy.
[9] Oliveira M, Henderson T (2003) What online gamers really think of the Internet?. In Proceedings of the 2nd Workshop on Network and system support for games (NetGames '03). ACM, New York, NY, USA, pp. 185-193.

[10] J. Saldana, J. Fernandez-Navajas, J. Ruiz-Mas, J. Murillo, E. Viruete, J. I. Aznar, "Evaluating the Influence of Multiplexing Schemes and Buffer Implementation on Perceived VolP Conversation Quality," Computer Networks (Elsevier), Volume 56, Issue 7, Pages 1893-1919, May 2012.

[11] A. F. Wattimena, R. E. Kooij, J. M. van Vugt, O. K. Ahmed, "Predicting the perceived quality of a first person shooter: the Quake IV G-model". In Proc. 5th SIGCOMM workshop Network and system support for games (NetGames '06), ACM, New York, NY, USA, 2006.

[12] M. Ries, P. Svoboda, M. Rupp, "Empirical study of subjective quality for Massive Multiplayer Games," Systems, Signals and Image Processing, 2008. IWSSIP 2008. 15th International Conference on, pp.181-184, 25-28, June 2008.

[13] F. Kuipers, R. Kooij, D. De Vleeschauwer, K. Brunnstrom, "Techniques for measuring Quality of Experience," Wired/Wireless Internet Com., Springer-Verlag Berlin/Heidelberg, pp. 216-227, 2010.

[14] J. Saldana, J. Fernandez-Navajas, J. Ruiz-Mas, E. Viruete Navarro, L. Casadesus, "Online FPS Games: Effect of Router Buffer and Multiplexing Techniques on Subjective Quality Estimators," Multimedia Tools and Applications, Springer, accepted for publication. doi 10.1007/s11042-012-1309-4

[15] S. Ratti, B. Hariri and S. Shirmohammadi, "A Survey of FirstPerson Shooter Gaming Traffic on the Internet," IEEE Internet Computing, vol 14, no. 5, pp. 60-69, 2010. 


\section{BIOGRAPHIES}

JOSE SALDANA (jsaldana@unizar.es) received his B.S. and M.S. in Telecommunications Engineering from University of Zaragoza, in 1998 and 2008, respectively. He received his Ph.D. degree in Information Technologies in 2011. He is currently a research fellow in the Department of Engineering and Communications of the same University. His research interests focus on Quality of Service in Realtime Multimedia Services, as VoIP and networked online games.

DAN WiNG (dwing@cisco.com) received his B.S. in Computer Science from Central Washington University in 1990, and is currently a Distinguished Engineer at Cisco where he has worked since 1996. He coauthored TCRTP (4170) which optimizes transmission of RTP streams by tunneling and compressing headers, and authored over a dozen RFCs and two dozen patents related to IPv6, Voice over IP, optimization, and voice security. He has co-chaired the IETF BEHAVE working group since 2006, which works on NAT64 standards. He is currently researching new technologies that provide user-visible benefits with IPv6 and improving co-existence of IPv6 and IPv4.

JULIÁN FERnÁNDEZ-NAVAJAS (navajas@unizar.es) received the Telecommunications Engineering degree from the Polytechnic University of Valencia, in 1993, and the Ph.D. degree from the University of Zaragoza, Spain, in 2000. He is currently an Associate Professor in the Centro Politécnico Superior, Universidad de Zaragoza. His professional research interests are in Quality of Service (QoS), Network Management, Telephony over IP, Mobile Networks, online gaming and other related topics.

JosÉ RUIz-MAS (jruiz@unizar.es) received the Engineering of Telecommunications degree from the Universitat Politècnica de Catalunya (UPC), Spain, in 1991 and the Ph.D. degree from the University of Zaragoza in 2001. He worked as a software engineer at the company TAO Open Systems from 1992 to 1994. In 1994 he joined the Centro Politécnico Superior as an Assistant Professor until 2003, when he became an Associate Professor. At present he is member of the Aragón Institute of Engineering Research (I3A) and his research activity lies in the area of Quality of Service in Multimedia Services with special emphasis on the provision of methodologies and tools to assess the perception of the end-user (Quality of Experience, QoE).

Muthu A. M. Perumal (mperumal@cisco.com) obtained his master's degree in computer science from Madurai Kamaraj University and a second master's degree in software engineering from Birla Institute of Technology \& Science (BITS), Pilani. He is currently a Technical Leader at Cisco and has years of experience working on VolP products such as gateways, IP PBXs and Session Border Controllers (SBCs). He is currently serving as the editor of the ITU-T specifications H.225.0 and H.323 Implementors' Guide. He co-authored the H.460.geo specification which describes how geo-location information is carried in $\mathrm{H} .323$ system. He has also co-authored several IETF drafts.

Michael Ramalho (mramalho@cisco.com) holds a B.S.E.E. from Rutgers University, a M.Eng.E.E. from Cornell University and a Ph.D. in speech signal processing from Rutgers University. He was Director of Internet Telephony research in Bellcore's Applied Research. During his tenure at Telcordia Technologies (successor to Bellcore) and Bell Laboratories, he held a variety of management and engineering positions. He was also Lead Telephony Technologist at Voxware, a Princeton, New Jersey based start-up. Currently, he is a video, speech, and audio signal processing architect in Cisco System's Collaboration Technology Group where he is involved in joint optimization of network transport technology and media signal processing technology. He is the inventor of a lossless compression algorithm for G.711, a successor of which became ITU-T Rec. G.711.0. His recent work includes designs for media over the Internet, seamless real-time IP mobility, IP transport designs (PR-SCTP/RFC 3758), IP transport diagnostics and media quality metrics. Dr. Ramalho is a Rutgers University CAIP Fellow, served on the Technical Program Committee of multiple IEEE Communications Society Conferences, has seventeen issued patents and is an IEEE Senior Member.

Gonzalo CAMARILlo (gonzalo.camarillo@ericsson.com) works for Ericsson Research in Finland as Principal Researcher in the area of Services and Software. In the past, he funded and headed the Advanced Multimedia Research Laboratory also at Ericsson Research Finland. He received a Ph.D. degree in Electrical Engineering from Aalto University (Helsinki, Finland). He also received M.Sc. degrees in
Electrical Engineering from the Stockholm (Sweden) Royal Institute of Technology and from Universidad Politecnica de Madrid (Spain). His research interests include signaling, multimedia applications, transport protocols, and networking architectures. He has authored a number of RFCs, books, patents, and scientific papers on these areas. Gonzalo has co-authored, among other standards, the SIP (Session Initiation Protocol) specification (RFC 3261). He has served on the IAB (Internet Architecture Board) and has chaired a number of IETF (Internet Engineering Task Force) working groups. Currently, Gonzalo is the director of the RAI (Real-time Applications and Infrastructures) area at the IETF. He is also the IETF liaison manager to 3GPP.

Fernando Pascual (fpb@tid.es) received the Telecommunications Engineering degree from the Alcalá de Henares University (UAH) in 2007, and the Master in Information Technologies and Communications, major in Computer Engineering and Networks, at the same university in 2011. He is working in Telefonica I+D since 2006 and his professional career is focused in the network intelligence and the M2M services development for the Telefonica group.

DIEGo R. LOPEZ (diego@tid.es) earned his MS in Physics from the University of Granada in 1985 and his PhD from the University of Seville in 2001. He is currently in charge of the Technology Exploration activities within the GCTO Unit of Telefónica I+D. After being one of the Telefónica I+D "founding fathers" back in the $80 \mathrm{~s}$, he worked for several years in the academic sector, as responsible for middleware infrastructures in RedIRIS (the Spanish NREN), before returning to Telefónica I+D in 2011. Diego is currently focused on identifying and evaluating new opportunities in technologies applicable to network infrastructures, and the coordination of national and international collaboration activities. His current research interests are related to network intelligence and virtualization, infrastructural services, and new network architectures.

MANUEL NUÑEz (mns@tid.es) received a degree in Telecommunication Engineering with the Polytechnic University of Madrid (Spain) in 1997. The same year he was employed as research engineer in Telefónica I+D and he is currently head of Network Automation \& Dynamization Group at Telefónica I+D. He is professor in the technical school of Telefónica and author or coauthor of several patents in control technologies and network signaling used in some Telefonica products. During these years he has been involved in a number of EU-funded projects and Telefonica Projects focusing on different aspects of network control, new generation networks, $\mathrm{M} 2 \mathrm{M}$ concepts, testbed and network architecture design.

DAVID FLoREz (dflorez@tid.es) obtained his Telecomunication Engineering degree from Madrid ETSIT in 1990. He works for Telefonica I+D since 1992 where he currently collaborates with the Network Dynamisation \& Optimisation Initiative in topics related with network control and QoS/QoE real time management.

JuAN A. CASTELl (jacl@tid.es) obtained his Technical engineering degree from the Politechnic University of Madrid (Spain) in 1991. After working as analyst-programmer in different companies always for telecommunications projects, his first requested task as Telefónica I+D employee was to implement its first AAA server for its IP network, Infoviat, in 1997. Since that moment until 2010 he has been responsible of the AAA and DNS implementations for Telefonica Spain IP services. Since 2010 is the technical coordinator of the Telefónica I+D innovation activities related with network intelligence and signaling/control protocols. He is also author or contributtor of several patents regarding these concepts.

TomASo DE CoLA (Tomaso.deCola@dlr.de) received the Laurea degree (with honors) in telecommunication engineering, in 2001, the Qualification degree as Professional Engineer in 2002, and the Ph. D. degree in Electronic and Computer Engineering, Robotics and Telecommunications in 2010 from the University of Genoa, Italy. From 2002 until 2007, he worked with the Italian Consortium of Telecommunications (CNIT), University of Genoa Research Unit, as a Scientist Researcher. Since 2008, he has been with the German Aerospace Centre (DLR), Wessling, Germany, where he is involved in different European Projects focusing on different aspects of DVB standards, CCSDS protocols, and testbed design. He is coauthor of more than 50 papers, including in international conferences and journals. His main research activity concerns: TCP/IP protocols, satellite networks, transport protocols for wireless links, interplanetary networks as well as delay tolerant networks. Dr. de Cola served on the Technical Program 
Committee at several IEEE International Conferences. He is a member of the IEEE Communications Society.

MATTEO BERIOLI (matteo.berioli@dlr.de) (Member, IEEE) received a Laurea degree (M.S.) in electronic engineering and the Ph.D. degree in information engineering from the University of Perugia, Perugia, Italy, both with honors, in 2001 and 2005, respectively. Since 2002, he has been with the German Aerospace Center (DLR), where since 2008 he has been leading the Networking and Protocols Group of the Digital Networks Department in the Institute of Communications and Navigation. His main research activities are in the area of IP-based satellite networks; key research issues include quality of service (QoS) and protocol analysis, cross-layer techniques, header compression, and packet-layer coding. Since 2006 he also has been working as expert for the European Telecommunications Standards Institute (ETSI) in the area of broadband satellite multimedia; from 2006 to 2008, he was the first chairman of the satellite working group of the Public Safety Communications Europe Forum (PSCE Forum). He is author/coauthor of more than 50 papers that appeared in international journals and conference proceedings. 